\title{
Quadratic Regularization Design for Fan Beam Transmission Tomography
}

\author{
Hugo Shi and Jeffrey A. Fessler \\ The University of Michigan, Ann Arbor, MI
}

\begin{abstract}
Statistical methods for tomographic image reconstruction have shown considerable potential for improving image quality in X-ray CT. Penalized-likelihood (PL) image reconstruction methods require maximizing an objective function that is based on the log-likelihood of the sinogram measurements and on a roughness penalty function to control noise. In transmission tomography, PL methods (and MAP methods) based on conventional quadratic regularization functions lead to nonuniform and anisotropic spatial resolution, even for idealized shift-invariant imaging systems. We have previously addressed this problem for parallel-beam emission tomography by designing data-dependent, shift-variant regularizers that improve resolution uniformity. This paper extends those methods to the fan-beam geometry used in X-ray CT imaging. Simulation results demonstrate that the new method for regularization design requires very modest computation and leads to nearly uniform and isotropic spatial resolution in the fan-beam geometry when using quadratic regularization.
\end{abstract}

Keywords: X-ray computed tomography $(\mathrm{CT})$, regularization, iterative image reconstruction, MAP, fan beam

\section{INTRODUCTION}

There is growing interest in the use of statistical image reconstruction methods for X-ray CT imaging due to the potential for reducing patient dose, reducing artifacts due to beam hardening and metal objects, and to accommodate scanning geometries that are poorly suited to conventional FBP reconstruction. Maximum likelihood methods for image reconstruction lead to excessively noisy images, so one must include some form of noise control, such as adopting a penalized-likelihood (PL) approach, or similarly a maximum a posteriori (MAP) method.

Although PL methods can control noise effectively, if one uses a conventional quadratic roughness penalty then the resulting reconstructed images will have nonuniform and anisotropic spatial resolution, even for idealized shift-invariant imaging systems, due to interactions between the non-quadratic Poisson log-likelihood and the regularization term [1]. For shift-variant systems, including the fan-beam geometry used in X-ray CT, there will be additional variation in spatial resolution over the field of view (FOV). This "problem" could be circumvented by using a conventional quadraticallypenalized, unweighted least-squares (QPULS) estimation method, but QPULS images have poor noise properties (akin to FBP in fact) because the weighting, which is explicit in PWLS methods [2] and implicit in penalized-likelihood methods, is a central advantage of statistical methods over FBP. We previously described a method for designing quadratic regularizers that improve the resolution uniformity and isotropy of reconstructed images for parallel-beam systems, focusing on emission tomography [3]. This paper addresses the fan-beam geometry for transmission tomography.

\section{SPATIAL RESOLUTION ANALYSIS AND REG. DESIGN}

We use two key concepts from previous work. One concept is local shift invariance [4-6]. Although fan-beam tomography systems are shift variant, the system and reconstruction method are often approximately shift invariant in the local neighborhood around any given pixel. This characteristic enables "local Fourier analysis" of the spatial resolution properties. The other concept is that for any given pixel, the effect of (implicit or explicit) ray-dependent weighting in a statistical reconstruction method can be approximated by one weight per projection view [6].

Hugo Shi: E-mail: hugoshi@eecs.umich.edu, Telephone: 734-255-3803

Jeffrey A. Fessler: E-mail: fessler@umich.edu, Telephone: 734-763-1434 


\subsection{Local impulse response}

One can quantify spatial resolution properties of image reconstruction methods using the local impulse response. Let $\boldsymbol{y}$ denote the projection measurement vector, $\boldsymbol{A}$ the system matrix, and $\boldsymbol{x}$ the unknown object vector (pixel values), where $\boldsymbol{y} \approx \boldsymbol{A x}$. For an estimator $\hat{\boldsymbol{x}}(\boldsymbol{y})$, we define the local impulse response for the $j$ th pixel to be

$$
\boldsymbol{l}^{j}=\lim _{\delta \rightarrow 0} \frac{\hat{\boldsymbol{x}}\left(\boldsymbol{y}+\delta \boldsymbol{A} \boldsymbol{e}_{j}\right)-\hat{\boldsymbol{x}}(\boldsymbol{y})}{\delta} .
$$

This PSF shows how a pertubation in the $j$ th pixel affects other pixels, and is our tool for regularization design.

\subsection{Regularized reconstruction}

Penalized likelihood reconstruction methods have the form

$$
\hat{\boldsymbol{x}}=\underset{\boldsymbol{x}}{\arg \min } L(\boldsymbol{A} \boldsymbol{x}, \boldsymbol{y})+R(\boldsymbol{x}),
$$

where $L$ denotes the negative log-likelihood and $R(\boldsymbol{x})$ denotes a roughness penalty function that regularizes the problem. We focus here on quadratic roughness penalties of the form $R(\boldsymbol{x})=\frac{1}{2} \boldsymbol{x}^{\prime} \boldsymbol{R} \boldsymbol{x}$, where $\boldsymbol{R}$ is the Hessian of the penalty function.

For such estimators, the local impulse response is $[1,7]$ :

$$
\boldsymbol{l}^{j}=\left[\boldsymbol{A}^{\prime} \nabla^{20} L(\boldsymbol{A} \hat{\boldsymbol{x}}, \boldsymbol{y}) \boldsymbol{A}+\boldsymbol{R}\right]^{-1} \boldsymbol{A}^{\prime}\left[-\nabla^{11} L(\boldsymbol{A} \hat{\boldsymbol{x}}, \boldsymbol{y})\right] \boldsymbol{A} \boldsymbol{e}_{j} .
$$

For typical log-likelihoods, this simplifies as follows [7]:

$$
\boldsymbol{l}^{j}=\left[\boldsymbol{A}^{\prime} \boldsymbol{W} \boldsymbol{A}+\boldsymbol{R}\right]^{-1} \boldsymbol{A}^{\prime} \boldsymbol{W} \boldsymbol{A} \boldsymbol{e}_{j},
$$

where $\boldsymbol{W}$ is a diagonal matrix that depends on the log-likelihood and $\boldsymbol{y}$. For transmission tomography with the usual Poisson model, $\boldsymbol{W} \approx \operatorname{diag}\left\{y_{i}\right\}$ [7].

\subsection{Regularization design}

Our goal is to choose the penalty coefficients $\boldsymbol{r}^{j}=\left(r_{1}, \ldots, r_{L}\right)$ (and hence $\boldsymbol{R}$ ) so that the local impulse response at each pixel $j$ closely matches some target PSF $\boldsymbol{l}_{0}$. Experience has shown that reasonable target PSFs have the form

$$
\boldsymbol{l}_{0}^{j}=\left[\boldsymbol{G}^{\prime} \boldsymbol{G}+\boldsymbol{R}_{0}\right]^{-1} \boldsymbol{G}^{\prime} \boldsymbol{G} \boldsymbol{e}_{j},
$$

where $\boldsymbol{G}$ denotes a shift-invariant system that approximates the possibly shift-varying system model $\boldsymbol{A}$, and $\boldsymbol{R}_{0}$ denotes a conventional shift-invariant regularizer. In other words, we would like to choose $\boldsymbol{R}$ (by choosing $\left\{\boldsymbol{r}^{j}\right\}$ ) such that

$$
\left[\boldsymbol{A}^{\prime} \boldsymbol{W} \boldsymbol{A}+\boldsymbol{R}\right]^{-1} \boldsymbol{A}^{\prime} \boldsymbol{W} \boldsymbol{A} \boldsymbol{e}_{j} \approx\left[\boldsymbol{G}^{\prime} \boldsymbol{G}+\boldsymbol{R}_{0}\right]^{-1} \boldsymbol{G}^{\prime} \boldsymbol{G} \boldsymbol{e}_{j}
$$

Achieving this goal should lead to nearly uniform and anisotropic spatial resolution.

Motivated by continuous-space analogs not shown, we "cross multiply," rearrange the matrices, and simplify, yielding

$$
R_{0} A^{\prime} W A e_{j} \approx R G^{\prime} G e_{j} .
$$

(Since all circulant matrices commute, matrices that are locally shift invariant will commute approximately.) Roughly speaking then, we would like to solve

$$
\min _{\left\{\boldsymbol{r}^{j}\right\}} \sum_{j}\left\|\boldsymbol{R}_{0} \boldsymbol{A}^{\prime} \boldsymbol{W} A \boldsymbol{e}_{j}-\boldsymbol{R} G^{\prime} \boldsymbol{G} \boldsymbol{e}_{j}\right\|
$$

where $\boldsymbol{R}$ depends on $\left\{\boldsymbol{r}^{j}\right\}$, for some type of norm. Instead of solving this minimization problem using matrix-vector operations, we follow the philosophy of [3] and replace all the matrices above with corresponding continuous-space Fourier domain expressions. We then solve for $\boldsymbol{r}^{j}$ analytically in the Fourier domain. 
In the above expressions, $G^{\prime} G$ is the cascade of forward and back-projection operations. In continuous-space, this ideally would correspond to convolution with $1 / r$ or multiplication by $1 / \rho$ in the Fourier domain, where $(\rho, \varphi)$ denotes polar coordinates in frequency space. A more reasonable model accounts at least for some "typical" detector response, such as in the following approximation:

$$
\boldsymbol{G}^{\prime} \boldsymbol{G} \equiv \frac{|B(\rho)|^{2}}{\rho},
$$

where $B(\cdot)$ denotes some user-selected frequency response, corresponding to the "typical" radial blur function, e.g., the blur at the center of a single projection view. For shift varying systems, let $b_{\varphi}^{j}(r)$ denote the detector response at angle $\varphi$ local to where the $j$ th pixel projects onto the detector at that angle. (For a shift-invariant system $b(r)$ would be independent of $\varphi$ and $j$.) Let $B_{\varphi}^{j}(\rho)$ denote the corresponding (local) frequency response. Similarly, let $w_{\varphi}(r)$ denote the diagonal "element" of $\boldsymbol{W}$ corresponding to angle $\varphi$ and radial position $r$, and define the following effective "certainty" at angle $\varphi$ for the $j$ th pixel ( $c f .[6$, eq. (19)]):

$$
w^{j}(\varphi) \triangleq \frac{\int_{-\infty}^{\infty}\left|b_{\varphi}^{j}(r)\right|^{2} w_{\varphi}(r) \mathrm{d} r}{\int_{-\infty}^{\infty}\left|b_{\varphi}^{j}(r)\right|^{2} \mathrm{~d} r} .
$$

Using continuous-space analogies, one can show

$$
\boldsymbol{A}^{\prime} \boldsymbol{W} \boldsymbol{A} \equiv \frac{w^{j}(\varphi)\left|B_{\varphi}^{j}(\rho)\right|^{2}}{\rho} .
$$

We refer to $\boldsymbol{A}^{\prime} \boldsymbol{W} \boldsymbol{A}$ as the Gram operator.

Previous work [3] continues from this point and leads to a regularizer design method that yields nearly uniform and isotropic spatial resolution for the parallel beam case. Here, we transform $w^{j}(\varphi)$ to fit a fan beam geometry. Then we can substitute our newly computed $w^{j}(\varphi)$ into the previous design method. This results in a corresponding regularizer that yields nearly uniform and isotropic spatial resolution for the fan beam case.

\section{FAN BEAM GEOMETRY}

Many contemporary tomographic imaging systems have fan beam geometries, including commercial X-ray CT scanners and some collimators for SPECT systems. For hypothetical continuous measurements, one could transform fan-beam projections into parallel-beam projections by a simple change of variables. For discrete, noisy measurements, rebinning fan-beam measurements into parallel-beam projections requires an interpolation operation that could degrade spatial resolution. To avoid such rebinning, one can derive reconstruction methods (and regularization methods) directly in terms of the fan-beam coordinates.

Fig. 1 illustrates the fan-beam geometry that will be considered here. Since it can be challenging mechanically to ensure that the line between the X-ray source and the midpoint of the detector passes through the exact center of rotation, we allow an offset $r_{\text {off }}$ between that line and the center [8]. Let $P$ denote the point along that line that intersects the circle of radius $r_{\text {off }}$ centered at the rotation isocenter. $D_{\mathrm{d}}$ denotes the distance from the point $P$ to the detector, $D_{\mathrm{s}}$ denotes the distance from the X-ray source to $P$, and $D_{\mathrm{f}}$ denotes the distance from the X-ray source to the focal point of the detector arc. Define $D_{\mathrm{c}} \triangleq D_{\mathrm{d}}+D_{\mathrm{s}}$ to be the total distance from the X-ray source to the center of the detector. This formulation allows the detector focal point to differ from the X-ray source location to encompass a variety of system configurations. For flat detectors, $D_{\mathrm{f}}=\infty$. For third-generation X-ray CT systems, $D_{\mathrm{f}}=0$. For fourth generation X-ray CT systems, $D_{\mathrm{f}}=-D_{\mathrm{s}}$.

In our notation, the distances $D_{\mathrm{d}}$ and $D_{\mathrm{s}}$ are constants, rather than being functions of angle $\beta$. Generalizations exist to allow non-circular source trajectories [9].

Let $s \in\left[-s_{\max }, s_{\max }\right]$ denote the (signed) arc length along the detector, with $s=0$ corresponding the detector center. Arc length is the natural parameterization for detector elements that are spaced equally along the detector. (For a flat detector with $D_{\mathrm{f}}=\infty$, the arc length $s$ is simply the position along the detector.) The various angles have the following relationships:

$$
\alpha(s)=\frac{s}{D_{\mathrm{c}}+D_{\mathrm{f}}}, \quad \gamma(s)=\tan ^{-1}\left(\frac{\left(D_{\mathrm{c}}+D_{\mathrm{f}}\right) \sin \alpha(s)}{\left(D_{\mathrm{c}}+D_{\mathrm{f}}\right) \cos \alpha(s)-D_{\mathrm{f}}}\right),
$$




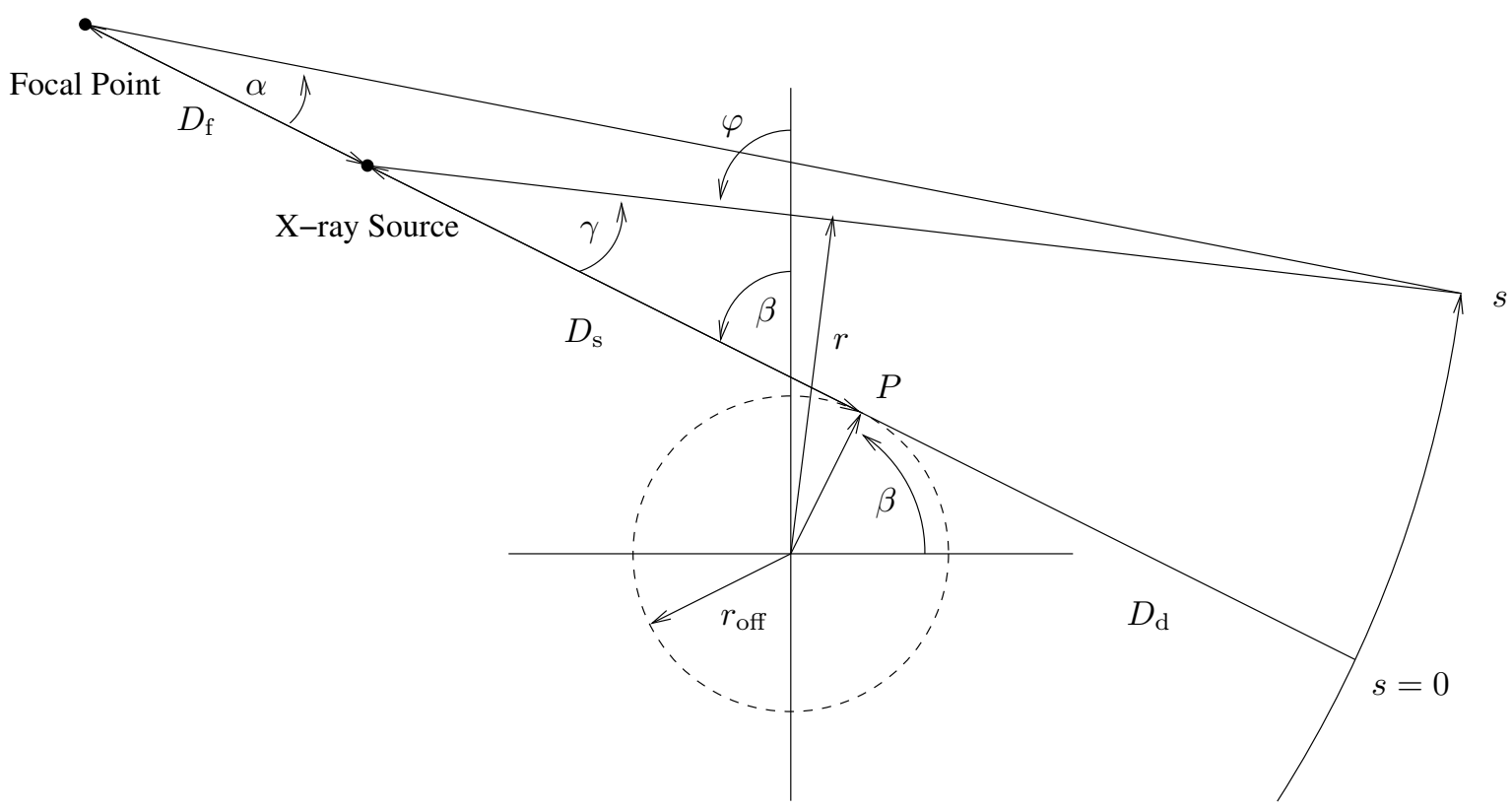

Figure 1. Illustration of fan beam geometry.

where the two most important cases are

$$
\tan \gamma(s)= \begin{cases}\tan \alpha(s), & D_{\mathrm{f}}=0 \\ s / D_{\mathrm{c}}, & D_{\mathrm{f}}=\infty\end{cases}
$$

The (inverse) relationship between $\gamma$ and $s$ is:

$$
s= \begin{cases}\left(D_{\mathrm{c}}+D_{\mathrm{f}}\right)\left[\gamma-\arcsin \left(\frac{D_{\mathrm{f}}}{D_{\mathrm{c}}+D_{\mathrm{f}}} \sin \gamma\right)\right], & 0 \leq D_{\mathrm{f}}<\infty \\ D_{\mathrm{c}} \tan \gamma, & D_{\mathrm{f}}=\infty .\end{cases}
$$

The ray corresponding to angle $\beta$ and detector element $s$ is

$$
\mathcal{L}(s, \beta)=\{(x, y): x \cos \varphi(s, \beta)+y \sin \varphi(s, \beta)=r(s)\},
$$

where

$$
\begin{aligned}
\varphi(s, \beta) & \triangleq \beta+\gamma(s) \\
r(s) & \triangleq D_{\mathrm{s}} \sin \gamma(s)+r_{\mathrm{off}} \cos \gamma(s) \\
& =\sqrt{D_{\mathrm{s}}^{2}+r_{\mathrm{off}}^{2}} \sin \left(\gamma(s)+\beta_{\mathrm{off}}\right),
\end{aligned}
$$

where we define $\beta_{\text {off }} \triangleq \angle\left(D_{\mathrm{s}}, r_{\text {off }}\right)$. The range of $r$ is limited inherently by the position of the X-ray source and the extent of the detector:

$$
|r(s)| \leq r_{\max } \triangleq D_{\mathrm{s}} \sin \gamma_{\max },
$$

where $\gamma_{\max } \triangleq \gamma\left(s_{\max }\right)$ and $s_{\max }$ is half of the total arc length of the detector. The radius $r_{\max }$ defines the circular field of view of the imaging system: the subset of the plane that is measured completely. (We assume that $D_{\mathrm{d}} \geq r_{\max }$, since otherwise even the detector center would limit the field of view.) The angle $2 \gamma_{\max }$ is called the fan angle. 
The line-integral projection $p(s, \beta)$ of $f$ along $\mathcal{L}(s, \beta)$ is*:

$$
\begin{aligned}
p(s, \beta) & =\int_{\mathcal{L}(s, \beta)} f(x, y) \mathrm{d} \ell \\
& =\iint f(x, y) \delta(x \cos \varphi(s, \beta)+y \sin \varphi(s, \beta)-r(s)) \mathrm{d} x \mathrm{~d} y,
\end{aligned}
$$

for $|s| \leq s_{\max }$ and $0 \leq \beta<\beta_{\max }$. We assume $\beta_{\max } \geq \pi+2 \gamma_{\max }$ to ensure complete sampling. The FBP reconstruction problem is to estimate $f$ from the fan-beam projections $\{p(s, \beta)\}$.

\section{ANALYSIS OF GRAM OPERATOR IN THE FAN BEAM CASE}

The usual inner product for fan-beam projection space is

$$
\left\langle p_{1}, p_{2}\right\rangle=\int_{-s_{\max }}^{s_{\max }} \int_{0}^{\beta_{\max }} p_{1}(s, \beta) p_{2}(s, \beta) \mathrm{d} s \mathrm{~d} \beta .
$$

This is the natural inner product when considering the usual case of samples that are equally-spaced in arc length $s$ and in source angle $\beta$. For this inner product, the adjoint of $\mathcal{P}$ is given by

$$
\left(\mathcal{P}^{*} p\right)(x, y)=\int_{-s_{\max }}^{s_{\max }} \int_{0}^{\beta_{\max }} \delta(x \cos \varphi(s, \beta)+y \sin \varphi(s, \beta)-r(s)) p(s, \beta) \mathrm{d} s \mathrm{~d} \beta,
$$

where $r(s)$ and $\varphi(s, \beta)$ were defined in (7).

Define a "diagonal" weighting operator $\mathcal{W}$ by

$$
(\mathcal{W} p)(s, \beta)=w(s, \beta) p(s, \beta),
$$

where $w(s, \beta)$ is a user-selected nonnegative weighting function. The natural QPWLS estimator for this continuous-space version of the image reconstruction problem has the form

$$
\hat{f}=\underset{f \in \mathcal{C}}{\arg \min }\|p-\mathcal{P} f\|_{\mathcal{W}^{1 / 2}}^{2}+R(f)
$$

where

$$
R(f)=\frac{1}{2}\langle f, \mathcal{R} f\rangle=\frac{1}{2}\left\|\mathcal{D}(\sqrt{R(\rho, \Phi)}) \mathcal{F}_{2} f\right\|^{2}
$$

and $\mathcal{C} \triangleq\left\{f \in \mathcal{L}_{2}\left(\mathbb{R}^{2}\right): \sqrt{x^{2}+y^{2}}>r_{\max } \Longrightarrow f(x, y)=0\right\}$, the subset of objects that are nonzero only within the field of view. (Without this restriction, it seems unlikely that the minimizer would be unique.)

We assume hereafter that $w(s, \beta)$ is chosen such that $w(s, \beta)=0$ when $\beta>\beta_{\max }$. Thus we can assume $\beta_{\max }=2 \pi$ for the analysis, yet the results are still applicable to "short" scans provided $w(s, \beta)$ is chosen appropriately. To analyze the impulse response of the Gram operator $\mathcal{P}^{*} \mathcal{W} \mathcal{P}$, consider an impulse object $\delta_{0}(x, y)=\delta\left(x-x_{0}, y-y_{0}\right)$ as follows:

$$
\begin{aligned}
& h\left(x, y ; x_{0}, y_{0}\right)=\left(\mathcal{P}^{*} \mathcal{W} \mathcal{P} \delta_{0}\right)(x, y) \\
&=\int_{0}^{2 \pi} \int_{-s_{\max }}^{s_{\max }} \delta(x \cos \varphi(s, \beta)+y \sin \varphi(s, \beta)-r(s)) \\
& \delta\left(x_{0} \cos \varphi(s, \beta)+y_{0} \sin \varphi(s, \beta)-r(s)\right) w(s, \beta) \mathrm{d} s \mathrm{~d} \beta .
\end{aligned}
$$

${ }^{*}$ Practically speaking, the integral should be restricted to the field of view: $\sqrt{x^{2}+y^{2}} \leq r_{\max }$, but this restriction would complicate analysis by introducing a shift variance into the problem, so we ignore it. 
For convenience, we express the point $\left(x_{0}, y_{0}\right)$ in polar coordinates $\left(r_{0}, \varphi_{0}\right)$. Now make the change of variables $r^{\prime}=$ $D_{\mathrm{s}} \sin \gamma(s), \varphi^{\prime}=\beta+\gamma(s)$ as defined in (7), assuming hereafter that $r_{\text {off }}=0$. Using the corresponding Jacobian determinant , the impulse response expression becomes

$$
\begin{aligned}
& h\left(x, y ; x_{0}, y_{0}\right)= \int_{0}^{2 \pi} \int_{-r_{\max }}^{r_{\max }} \delta\left(x \cos \varphi^{\prime}+y \sin \varphi^{\prime}-r^{\prime}\right) \\
& \cdot \delta\left(r_{0} \cos \left(\varphi^{\prime}-\varphi_{0}\right)-r^{\prime}\right) w\left(s^{\prime}, \beta^{\prime}\right) J\left(s^{\prime}\right) \mathrm{d} r^{\prime} \mathrm{d} \varphi^{\prime} \\
&=\int_{0}^{2 \pi} \delta\left(x \cos \varphi^{\prime}+y \sin \varphi^{\prime}-r_{0}^{\prime}\right) w\left(s^{\prime}, \beta^{\prime}\right) J\left(s^{\prime}\right) 1_{\left\{\left|r_{0}^{\prime}\right| \leq r_{\max }\right\}} \mathrm{d} \varphi^{\prime},
\end{aligned}
$$

where, using (6) and (7):

$$
\begin{aligned}
r_{0}^{\prime} & =r_{0} \cos \left(\varphi^{\prime}-\varphi_{0}\right) \\
s^{\prime} & =\left(D_{\mathrm{c}}+D_{\mathrm{f}}\right)\left[\arcsin \left(\frac{r_{0}^{\prime}}{D_{\mathrm{s}}}\right)-\arcsin \left(\frac{D_{\mathrm{f}}}{D_{\mathrm{c}}+D_{\mathrm{f}}} \frac{r_{0}^{\prime}}{D_{\mathrm{s}}}\right)\right] \\
\beta^{\prime} & =\varphi^{\prime}-\arcsin \left(\frac{r_{0}^{\prime}}{D_{\mathrm{s}}}\right) .
\end{aligned}
$$

Hereafter we focus on points within the FOV where $r_{0} \leq r_{\max }$. In the spirit of local shift invariance, consider the following local impulse response

$$
\begin{aligned}
h_{0}(r, \varphi) & \triangleq h\left(x_{0}+r \cos \varphi, y_{0}+r \sin \varphi ; x_{0}, y_{0}\right) \\
& =\int_{0}^{2 \pi} \delta\left(r \cos \left(\varphi-\varphi^{\prime}\right)\right) w\left(s^{\prime}, \beta^{\prime}\right) J\left(s^{\prime}\right) \mathrm{d} \varphi^{\prime} \\
& =\frac{1}{|r|}\left[\left.w\left(s^{\prime}, \beta^{\prime}\right) J\left(s^{\prime}\right)\right|_{\varphi^{\prime}=\varphi+\pi / 2}+\left.w\left(s^{\prime}, \beta^{\prime}\right) J\left(s^{\prime}\right)\right|_{\varphi^{\prime}=\varphi-\pi / 2}\right] .
\end{aligned}
$$

Thus, similar to the parallel-beam case, for the fan-beam case the local impulse response of the Gram operator is

$$
h_{0}(r, \varphi)=\frac{1}{|r|} w_{0}(\varphi+\pi / 2)
$$

where the angular-dependent weighting is

$$
\left.w_{0}(\varphi) \triangleq w\left(s^{\prime}, \beta^{\prime}\right) J\left(s^{\prime}\right)\right|_{\varphi^{\prime}=\varphi}+\left.w\left(s^{\prime}, \beta^{\prime}\right) J\left(s^{\prime}\right)\right|_{\varphi^{\prime}=\varphi-\pi} .
$$

It follows then, that the the local frequency response of the Gram operator is

$$
H_{0}(\rho, \Phi)=\frac{1}{|\rho|} w_{0}(\Phi)
$$

It is interesting that the local impulse response and frequency response have the same form in the fan-beam and parallelbeam cases.

In the equiangular case, where $D_{\mathrm{f}}=0$, we have the following simplifications:

$$
\begin{aligned}
r_{0}^{\prime} & = \pm r_{0} \cos \left(\varphi-\varphi_{0}\right) \\
s^{\prime} & = \pm D_{\mathrm{c}} \arcsin \left(\frac{r_{0} \cos \left(\varphi-\varphi_{0}\right)}{D_{\mathrm{s}}}\right) \\
\beta^{\prime} & = \begin{cases}\varphi-\arcsin \left(\frac{r_{0} \cos \left(\varphi-\varphi_{0}\right)}{D_{\mathrm{s}}}\right), & r_{0}^{\prime}=+r_{0} \cos \left(\varphi-\varphi_{0}\right) \\
\varphi-\pi+\arcsin \left(\frac{r_{0} \cos \left(\varphi-\varphi_{0}\right)}{D_{\mathrm{s}}}\right), & r_{0}^{\prime}=-r_{0} \cos \left(\varphi-\varphi_{0}\right)\end{cases} \\
J\left(s^{\prime}\right) & =\frac{D_{\mathrm{s}}}{D_{\mathrm{c}} \sqrt{1-\left(\frac{r_{0}}{D_{\mathrm{s}}}\right)^{2} \cos ^{2}\left(\varphi-\varphi_{0}\right)},}
\end{aligned}
$$

from which we can compute $w_{0}(\varphi)$ easily using (13). 


\section{SIMULATION RESULTS}

We simulate a 2D fan-beam CT system that images a $240 \mathrm{mmx} 240 \mathrm{~mm}$ area. The center of our object is $408.075 \mathrm{~mm}$ from the detector, and the source is $949.075 \mathrm{~mm}$ from the detector. The axis of rotation is at the center of the object. The simulated imaging system has 280 rays per view spaced $4 \mathrm{~mm}$ apart, and 100 evenly spaced view angles over a full $2 \pi$ rotation. The reconstructed images consisted of a $120 \times 120$ grid of $2 \mathrm{~mm}$ pixels.

We chose a target spatial resolution of 2.6 pixels or equivalently $5.2 \mathrm{~mm}$. Fig. 2 and Fig. 3 show the ideal local impulse response, the local impulse response for a conventional regularization method, and the local impulse response that results from regularization with our proposed Fourier-based design method for a pixel located at an offset of $(20,10)$ and $(-15,-15)$ pixels respectively from the center of the image. As is clear from the figure, the proposed regularization scheme yields more isotropic impulse responses.

The penalty coefficients yielding these results are shown in Fig. 4. The top images show coefficients in the horizontal and vertical neighbors, and the bottom images show coefficients for diagonal neighbors.

We simulated a noiseless fan-beam sinogram using the true image $\boldsymbol{x}$ shown in Fig. 5. We reconstructed images $\hat{\boldsymbol{x}}$ using three methods. Fig. 5 shows the image resulting from unweighted reconstruction (QPULS), conventional regularized weighted least squares reconstruction and our Fourier based regularized reconstruction. One can see that the rings in the conventionally reconstructed image have less uniform brightness than the image reconstructed by our proposed method. Fig. 6 shows profiles around the rings of the reconstructed images, verifying that our proposed scheme improves resoluton uniformity.
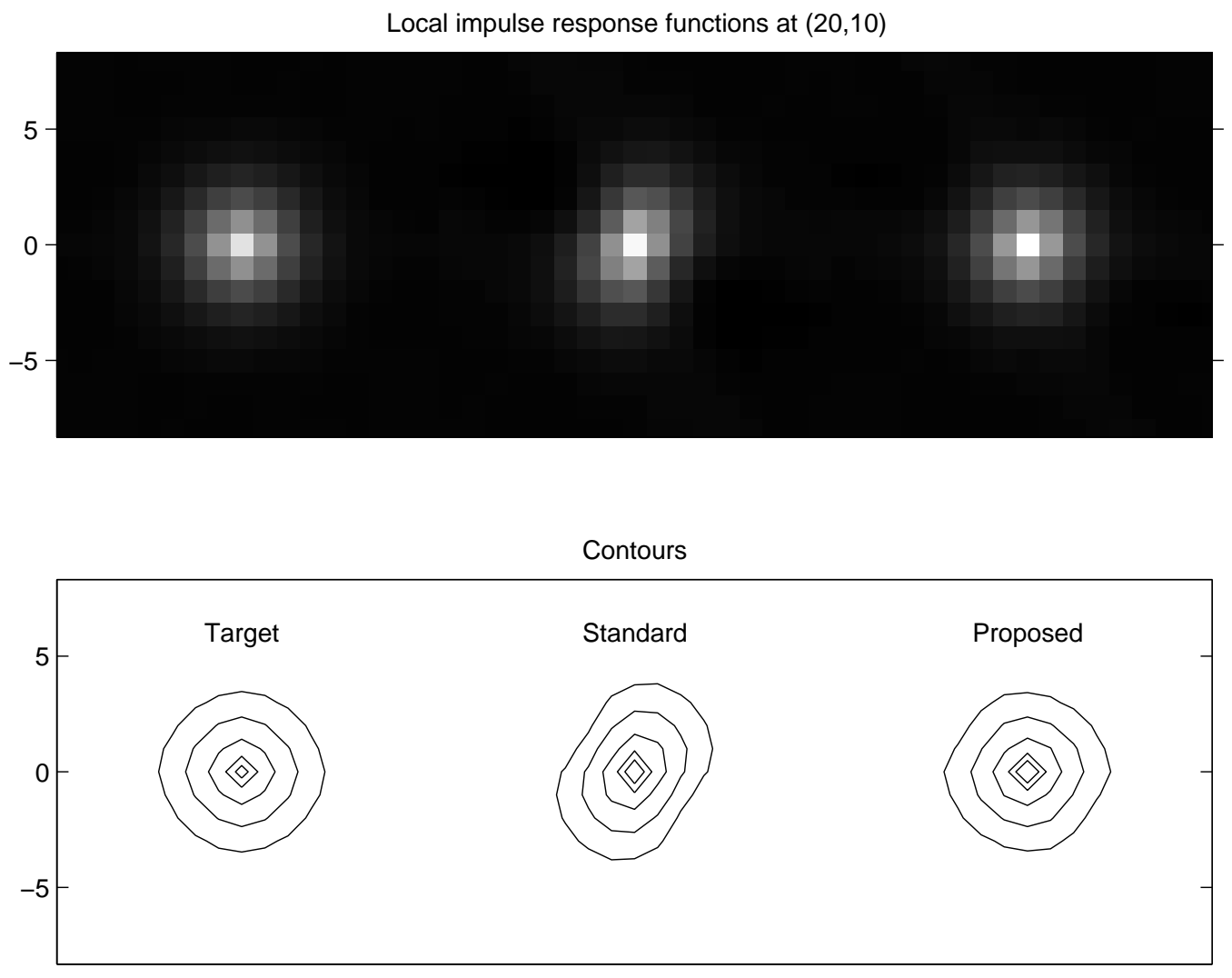

Figure 2. impulse responses

\section{SUMMARY}

This paper has summarized an extension of previous Fourier-based regularization design [3] to the fan-beam case. This extension is suprisingly simple and relatively easy to compute. Simulation results show that the method improves isotropy 

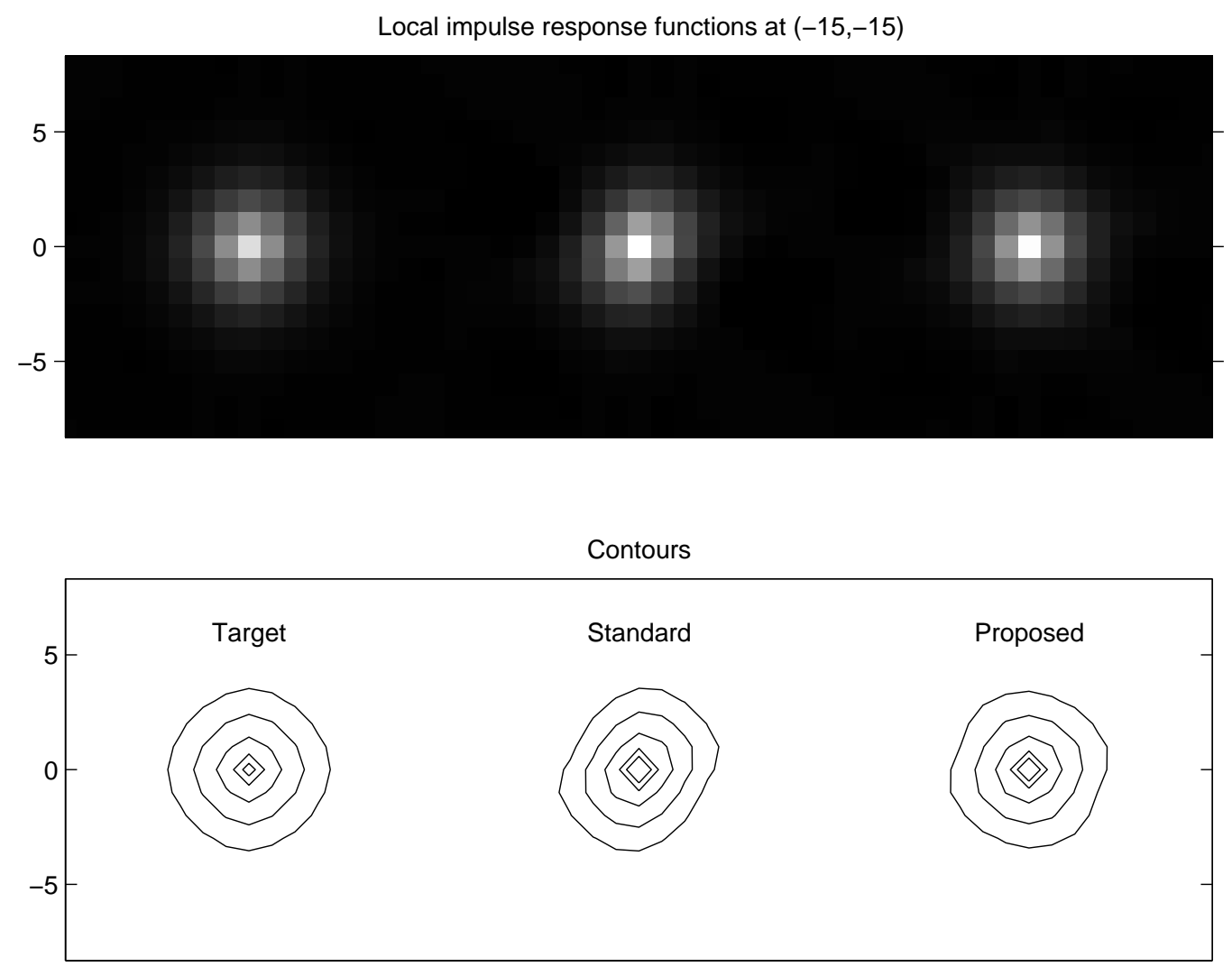

Figure 3. impulse responses

and uniformity of spatial resolution properties compared to conventional quadratic regularization schemes. Future work includes generalization to spatially variant blur, 3D extensions to cone beam CT, and application to real X-ray CT data.

\section{ACKNOWLEDGMENTS}

Work supported in part by NSF grant BES-9982349, NIH grants CA-60711 and CA-87634, and DOE grant DE-FG0287ER60561.

\section{REFERENCES}

1. J. A. Fessler and W. L. Rogers, "Spatial resolution properties of penalized-likelihood image reconstruction methods: Space-invariant tomographs," IEEE Tr. Im. Proc. 5, pp. 1346-58, Sept. 1996.

2. J. A. Fessler, "Penalized weighted least-squares image reconstruction for positron emission tomography," IEEE Tr. Med. Imag. 13, pp. 290-300, June 1994.

3. J. A. Fessler, "Analytical approach to regularization design for isotropic spatial resolution," in Proc. IEEE Nuc. Sci. Symp. Med. Im. Conf., 3, pp. 2022-6, 2003.

4. J. A. Fessler and S. D. Booth, "Conjugate-gradient preconditioning methods for shift-variant PET image reconstruction,” IEEE Tr. Im. Proc. 8, pp. 688-99, May 1999.

5. J. Qi and R. M. Leahy, "A theoretical study of the contrast recovery and variance of MAP reconstructions with applications to the selection of smoothing parameters," IEEE Tr. Med. Imag. 18, pp. 293-305, Apr. 1999.

6. J. W. Stayman and J. A. Fessler, "Regularization for uniform spatial resolution properties in penalized-likelihood image reconstruction," IEEE Tr. Med. Imag. 19, pp. 601-15, June 2000.

7. J. W. Stayman and J. A. Fessler, "Compensation for nonuniform resolution using penalized-likelihood reconstruction in space-variant imaging systems," IEEE Tr. Med. Imag. 23, pp. 269-84, Mar. 2004. 


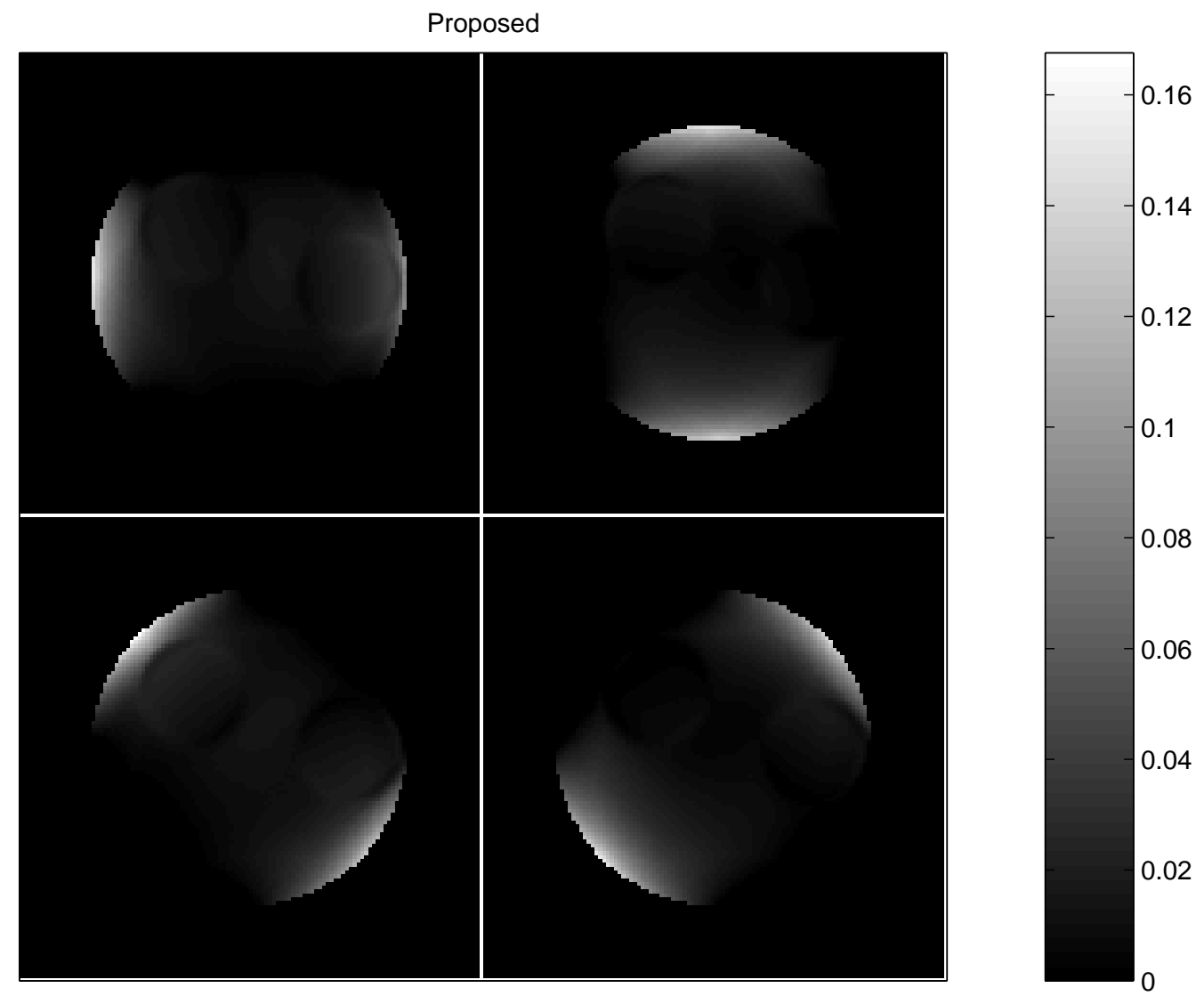

Figure 4. penalty coefficients

8. G. T. Gullberg, C. R. Crawford, and B. M. W. Tsui, "Reconstruction algorithm for fan beam with a displaced centerof-rotation," IEEE Tr. Med. Imag. 5, pp. 23-9, Mar. 1986.

9. G. Besson, "CT fan-beam parametrizations leading to shift-invariant filtering," Inverse Prob. 12, pp. 815-33, Dec. 1996. 

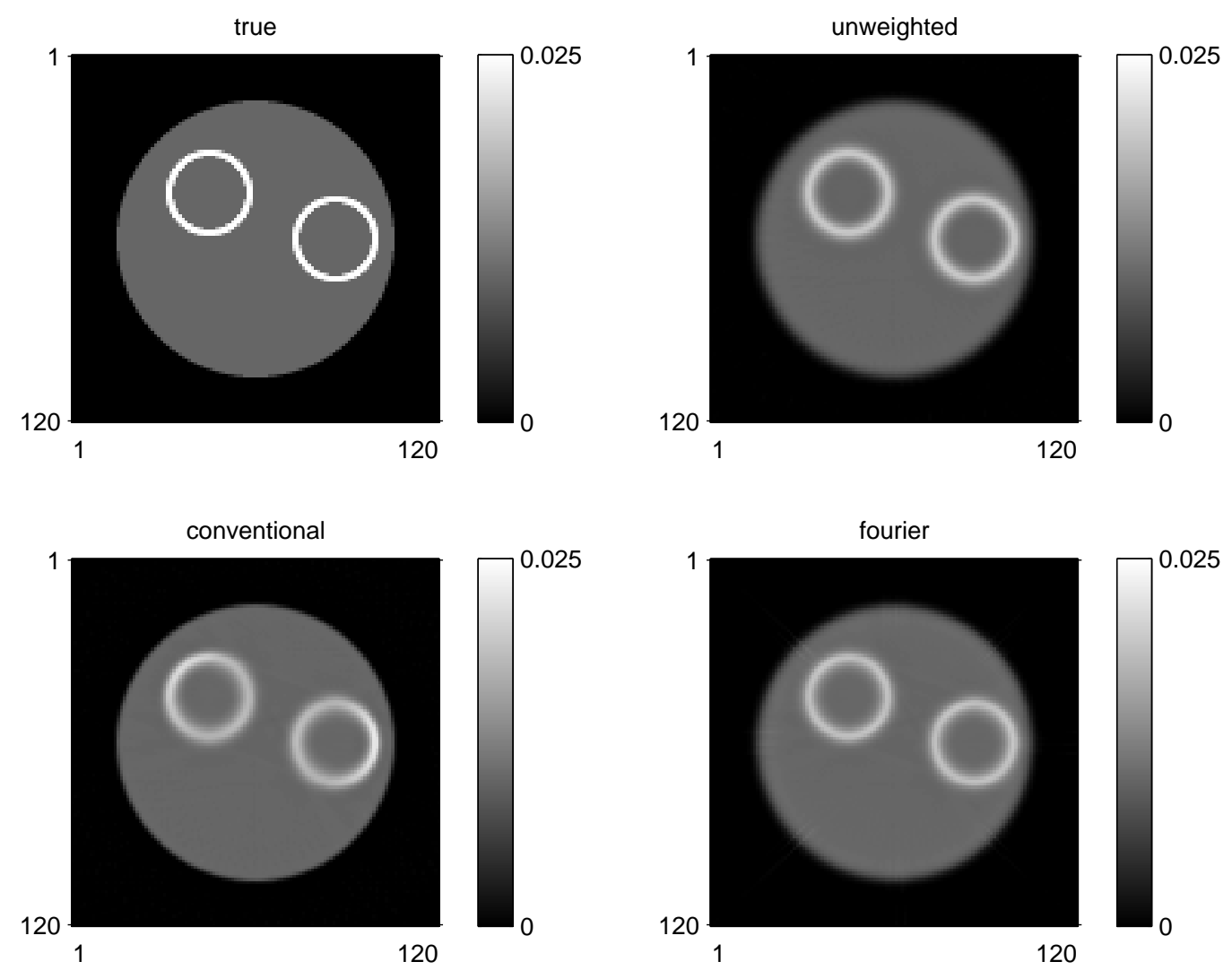

Figure 5. reconstructed images 


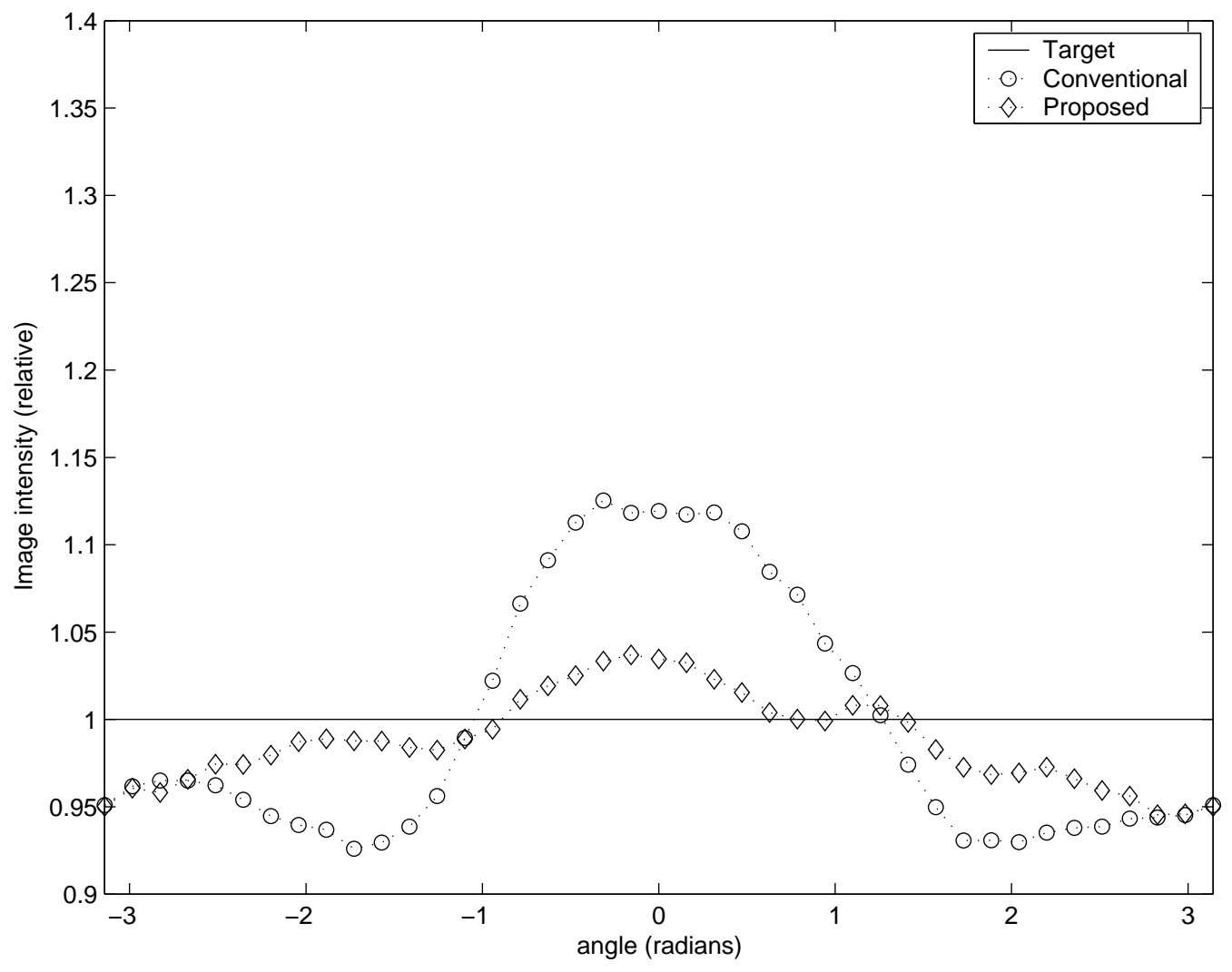

Figure 6. ring profiles 